\title{
Curcumin Enhances the Radiosensitivity of U87 Cells by Inducing DUSP-2 Up- Regulation
}

Yu Qian Jianfen Ma Xiaoyi Guo Jun Sun Yongtao Yu Boqiang Cao Lei Zhang Xiaopeng Ding Jin Huang Jun Fei Shao

Department of Neurosurgery, Wuxi People's Hospital, Nanjing Medical University, Wuxi, China

\section{Retracted Statement See Next Page}




\section{Retraction Statement}

Paper by Qian Y, Ma J, Guo X, Sun J, Yu Y, Cao $B$, Zhang L, Ding $X$, Huang J, Shao JF, entitled 'Curcumin Enhances the Radiosensitivity of U87Cells byInducing DUSP-2Up-Regulation' [Cell Physiol Biochem 2015;35(4):1381-93. doi: 10.1159/000373959]

Due to some important errors in this paper, we cannot repeat some critical results in our recent studies. To avoid any negative impact on other's research in this field, after serious consideration, we retract this paper.

We sincerely appreciate the efforts of the publishers, editors and reviewers and apologize for our decision.

Jun-fei Shao

Wuxi, Jiangsu Province, China 